\title{
A NOTE ON FREE PRODUCTS OF LINEAR GROUPS
}

\author{
ZBIGNIEW S. MARCINIAK
}

\begin{abstract}
For a field $K$, let $\bar{K}$ denote its algebraic closure. Assume that $|\bar{K}: K|=\infty$. Then for any linear groups $G, H \subseteq \mathrm{GL}_{n}(K)$ their free product $G * H$ can be embedded into $\mathrm{GL}_{N}(K(t))$. Here $N$ is an integer depending on $K$ only and $t$ stands for an indeterminate.
\end{abstract}

If $K$ is a field, let $\bar{K}$ denote its algebraic closure. We are going to prove

THEOREM. Let $G, H \subset \mathrm{GL}_{n}(K)$ be subgroups such that all entries of matrices $g \in G, h \in H$ lie in a subfield $K_{0} \subseteq K$ with $\left|\bar{K}_{0}: K_{0}\right|=\infty$. Then the free product $G * H$ can be embedded into $\mathrm{GL}_{N}(K(t))$. Here $t$ is an indeterminate and $N$ is an integer depending on $K_{0}$ and $n$ only.

We need two lemmata.

LEMMA A. Let $L$ be a field satisfying $|\bar{L}: L|=\infty$. For any integer $m$ there exists $a \in \bar{L}$ such that $\operatorname{deg}_{L} a>m$.

ProOF. If $\bar{L}$ is separable over $L$ it follows from the Theorem of Primitive Element [1, Chapter VII, $\S 6$, Theorem 14, p. 185]. If not, i.e. if $L$ is not perfect, say $a \in L \backslash L^{p}$, then the elements $a^{1 / p}, a^{1 / p^{2}}, \ldots$ have degrees $p, p^{2}, \ldots$ over $L$.

LEMMA B. Let $L$ be a field satisfying $|\bar{L}: L|=\infty$. If $S \subseteq \mathrm{GL}_{n}(L)$ is a subgroup with $S \cap L \cdot 1_{n}=\left\{1_{n}\right\}$, then there exists an embedding $S \subseteq \mathrm{GL}_{n}(\tilde{L})$ such that

(i) $|\tilde{L}: L|<\infty$,

(ii) for any $s \in S \backslash\{1\}, s_{i j} \neq 0$ for all $i, j$.

ProOF. Choose a matrix $x=\left(x_{i j}\right)$ of indeterminates and consider $x S x^{-1} \subseteq$ $\mathrm{GL}_{n}\left(L\left(x_{i j}\right)\right)$. Since any $s \in S \backslash\{1\}$ is a nonscalar matrix we get by [3, Theorem 2.13] that all entries of $x s x^{-1}$ are nontrivial elements of $L\left(x_{i j}\right)$. Moreover, each entry of $x s x^{-1}$ is of the form $p\left(x_{i j}\right) / \operatorname{det} x$ where $p$ is a polynomial of degree not exceeding $n$. In particular,

$$
x S x^{-1} \subseteq \mathrm{GL}_{n}\left(L\left[x_{i j},(\operatorname{det} x)^{-1}\right]\right) .
$$

Now, applying Lemma $\mathrm{A} n^{2}$ times, we find a sequence of simple algebraic extensions, each of degree $>n$ over the previous field

$$
L \subseteq L\left(a_{11}\right) \subseteq L\left(a_{11}, a_{12}\right) \subseteq \cdots \subseteq L\left(a_{11}, \ldots, a_{n n}\right)=\tilde{L} .
$$

Before we conclude the proof let us make a

REMARK. If $p \in L\left[x_{i j}\right]$ is a nonzero polynomial and $\operatorname{deg} p \leq n$, then $p\left(a_{i j}\right) \neq 0$. It is clear from the construction of $\tilde{L}$.

Received by the editors July 19, 1983.

1980 Mathematics Subject Classification. Primary 20G15; Secondary 20E06.

(C) 1985 American Mathematical Society $0002-9939 / 85 \$ 1.00+\$ .25$ per page 
Consider the $L$-algebra homomorphism $\phi: L\left[x_{i j}\right] \rightarrow \tilde{L}$ defined by $\phi\left(x_{i j}\right)=a_{i j}$. It factors to

$$
\phi: L\left[x_{i j},(\operatorname{det} x)^{-1}\right] \rightarrow \tilde{L}
$$

as $\operatorname{deg} \operatorname{det}=n$ (see Remark). It defines

$$
\phi_{*}: \mathrm{GL}_{n}\left(L\left[x_{i j},(\operatorname{det} x)^{-1}\right]\right) \rightarrow \mathrm{GL}_{n}(\tilde{L}) .
$$

From the Remark it follows that each entry of $x s x^{-1}$ is mapped by $\phi$ onto a nonzero element, so in particular $\phi_{*} \mid S$ is an isomorphism. Thus $\phi_{*}(S) \subseteq \mathrm{GL}_{n}(\tilde{L})$ is the required embedding.

PROOF OF THE THEOREM. Let $G, H \subseteq \mathrm{GL}_{n}\left(K_{0}\right)$ be subgroups with $K_{0} \subseteq$ $K,\left|\bar{K}_{0}: K_{0}\right|=\infty$. Let $\rho_{+}, \rho_{-}$denote the natural embeddings $\mathrm{GL}_{n}\left(K_{0}\right) \rightarrow$ $\mathrm{GL}_{2 n}\left(K_{0}\right)$,

$$
\rho_{+}(a)=\left[\begin{array}{cc}
a & 0 \\
0 & 1_{n}
\end{array}\right], \quad \rho_{-}(a)=\left[\begin{array}{cc}
1_{n} & 0 \\
0 & a
\end{array}\right] .
$$

Then $G \cong \rho_{+}(G), H \cong \rho_{-}(H) \subseteq \mathrm{GL}_{2 n}\left(K_{0}\right)$ and $H \cap G=\left\{1_{2 n}\right\}$.

Let $S=\langle G, H\rangle \subseteq \mathrm{GL}_{2 n}\left(K_{0}\right)$ be the subgroup generated by $G \cup H$. Consider the image of $S$ under the embedding $\mathrm{GL}_{2 n}\left(K_{0}\right) \subseteq \mathrm{GL}_{2 n+1}\left(K_{0}\right), a \mapsto\left[\begin{array}{ll}a & 0 \\ 0 & 1\end{array}\right]$. Then $S \cap K_{0} \cdot 1_{2 n+1}=\left\{1_{2 n+1}\right\}$.

By Lemma B, we can embed $S$ into $\mathrm{GL}_{2 n+1}\left(\tilde{K}_{0}\right)$ so that

(i) $\left|\tilde{K}_{0}: K_{0}\right|<\infty$,

(ii) for $s \in S \backslash\{1\}$, all $s_{i j} \neq 0$.

This gives in particular embeddings $G, H \subseteq S \subseteq \mathrm{GL}_{2 n+1}\left(\tilde{K}_{0}\right)$ with

(i) $G \cap H=\{1\}$,

(ii) $g \in G \backslash\{1\} \Rightarrow g_{1,2 n+1} \neq 0$,

(iii) $h \in H \backslash\{1\} \Rightarrow h_{2 n+1,1} \neq 0$.

These are precisely the assumptions of Shalen's Theorem [2, Proposition 1.3]. It follows that the map

defined by

$$
\rho: G * H \rightarrow \mathrm{GL}_{2 n+1}\left(\tilde{K}_{0}\left[t, t^{-1}\right]\right)
$$

$G \ni g \mapsto\left[\begin{array}{cccc}t & & & 0 \\ & t^{2} & & \\ & \ddots & \\ 0 & & & t^{2 n+1}\end{array}\right] \cdot g \cdot\left[\begin{array}{cccc}t^{-1} & & & 0 \\ & t^{-2} & & \\ & & \ddots & \\ 0 & & & t^{-(2 n+1)}\end{array}\right], \quad H \ni h \mapsto h$,

has the following property:

If $a=\rho\left(g_{1}\right) \rho\left(h_{1}\right) \cdots \rho\left(g_{r}\right) \rho\left(h_{r}\right)$ and all $g_{i}, h_{i} \neq 1$, then Trace $(a)=$ $\alpha \cdot t^{2 n r}+$ lower (possibly negative) powers of $t ; \alpha \neq 0$.

Clearly, $\rho$ is an embedding of $G \cup H$. Further, each element $w$ of $G * H \backslash(G \cup H)$ is conjugate to one in the form $g_{1} h_{1} \cdots g_{r} h_{r}$ with $g_{i} \in G \backslash\{1\}, h_{i} \in H \backslash\{1\}$. Therefore, Trace $\rho(w) \neq$ Trace $\rho(1)$, hence $\rho(w) \neq 1$. Thus $\rho$ embeds $G * H$ into $\operatorname{GL}_{2 n+1}\left(\tilde{K}_{0}(t)\right)$. To finish the proof, notice that $\tilde{K}_{0}(t)=K_{0}(t)\left(a_{i j}\right)$ and so

Therefore,

$$
\left|\tilde{K}_{0}(t): K_{0}(t)\right|=d<\infty \text {. }
$$

$$
G * H \subseteq \mathrm{GL}_{(2 n+1) d}\left(K_{0}(t)\right) \subseteq \mathrm{GL}_{(2 n+1) d}(K(t))
$$


REMARK. The value of $N=(2 n+1) d$ depends on the set of integers occurring as degrees of elements of $\bar{K}_{0}$ over $K_{0}$. In particular, if $K_{0}$ has irreducible polynomials of all degrees we can take $N=(2 n+1) \cdot(2 n+2)^{(2 n+1)^{2}}$, as easily follows from $[1$, p. 223]. I am indebted to the referee for this remark.

COROLLARY 1. Let $K$ be a field such that $|\bar{K}: K|=\infty$ (which is equivalent to $\bar{K} \neq K(\sqrt{-1})$, see $[1, p .223$, Corollary 2$])$. For any groups $G, H \subseteq \mathrm{GL}_{n}(K), G *$ $H \subseteq \mathrm{GL}_{N}(K(t))$.

COROLLARY 2. If $G, H$ are finitely generated groups, $K$ any field and $G, H \subseteq$ $\mathrm{GL}_{n}(K)$, then $G * H \subseteq \mathrm{GL}_{N}(K(t))$.

ProOF. Notice that an extension $K_{0}$ of a prime field by a finite set satisfies $\left|\bar{K}_{0}: K_{0}\right|=\infty$.

\section{REFERENCES}

1. S. Lang, Algebra, Addison-Wesley, Reading, Mass., 1965.

2. P. B. Shalen, Linear representations of amalgamated products, J. Pure Appl. Algebra 15 (1979), 87-97.

3. B. A. F. Wehrfritz, Infinite linear groups, Queen Mary College, London, 1969.

Institute of MAThematics, WARSAW UNiversity, 00-901 WARSAW, Poland, PKIN IXP 